\title{
Gentrificación de escala intermedia global en Latinoamérica. El caso de la reconstrucción de Managua, Nicaragua 1972-2014
}

Gentrification at a medium global scale in Latin America. The case of the reconstruction of Managua, Nicaragua 1972 - 2014

Dr. Jorge Inzulza Contardo, Néstor Saúl López Irías

\section{Filiación}

Universidad de Chile, Universidad Nacional de Nicaragua

E mail: jinzulza@uchilefau.cl,nlopez84@hotmail.com

\section{Resumen}

Hoy en día, el estudio de la gentrificación se posiciona como un proceso posible de aplicar en prácticamente toda la escala del territorio global. Dentro de este contexto, el rol que cumplen las ciudades intermedias es crucial y, sobretodo, aquellas que se ven afectas a procesos de reconstrucción post terremotos, como es el caso de América Latina. El presente artículo amplifica el debate de la gentrificación en el contexto de las políticas urbanas post terremotos aplicadas en ciudades intermedias, analizado bajo el marco-teórico de Diagrama de Ciclo de Alerta del Terremoto (DCAT). Se documenta el proceso de gentrificación producido por las intervenciones urbanas en la antigua área central de la ciudad Managua, Nicaragua entre los años 1972 y 2014.Se relevan cinco hitos historiográficos que dicen relación con políticas específicas de reconstrucción, que estarían facilitando la especulación de suelo urbano de la ciudad, generando por una parte, variables de reemplazo social y, por otra, extendiendo el área urbana y su relación metropolitana-intermedia. El artículo concluye que la gentrificación ha estado presente desde el inicio de las intervenciones post 1972 en esta ciudad, sin embargo, no es parte de la agenda gubernamental, requiriendo entonces, de mayor visibilidad y reversión de este proceso como oportunidad de desarrollo urbano.

\section{Palabras claves}

Gentrificación; Reconstrucción; Managua; Ciudad intermedia; Latinoamérica

\begin{abstract}
In the contemporary context, it is possible to study gentrification processes at a wide range of scales across the global territory. Within this framework, the role of intermediate cities is crucial, especially when undergoing post-earthquake reconstruction, as it happens in many Latin American cities. This article amplifies the gentrification debate, looking at reconstruction urban policies applied on intermediate cities, using the Diagram of Earthquake Alert Cycle (DCAT) as theoretical framework. The gentrification process triggered by interventions in Managua's central area, between 1972 and 2014, is documented. Five landmarks related to specific reconstruction policies, are studied, as they have played a role in the development of real estate speculation processes, contributing to social replacement, as well as the growth of the urban area of the city. The article concludes that gentrification has been present since the beginning of the post 1972 interventions in the city of Managua, even though it is not regarded as part of the government's agenda, which means that it requires attention, in order to revert the process towards the generation of new opportunities for urban development.
\end{abstract}

Key words

Gentrification; Reconstruction; Managua; Intermediate city; Latin America 


\section{Sumario}

Introducción

1 Cambio socio espacial en las ciudades intermedias

1.1 Aceleración en el contexto post terremoto

1.2 Diagrama de Ciclo de Alerta del Terremoto (DCAT)

2 Managua: una ciudad metropolitana con rol intermedio

2.1 El terremoto del 23 diciembre 1972: inicio de la transformación socio espacial

2.2 Periodo 1973-1978: política urbana en el contexto de estrategias de post-terremoto

2.3 Periodo 1979-1990: implicaciones de políticas sociales

2.4 Periodo 1991-2006: crecimiento del sector inmobiliario

2.5 Periodo 2007-2014: expansión del área metropolitana y nueva política de vivienda

3 Los "planes en papel" frente al proceso de gentrificación en Managua

Conclusión

Agradecimientos

Referencias

\section{Introducción}

El estudio de la gentrificación hoy en día se posiciona como un proceso posible de aplicar en prácticamente toda la escala del territorio global. Un análisis de las tendencias de este proceso muestra una clara mutación como es definida por Lees et al. (2008), de un asunto más esporádico en su origen (Islington, Londres), hacia un proceso que en la actualidad abarca barrios y áreas urbanas de mayor tamaño, llegando incluso a observarse en el ámbito rural. Este proceso no es la excepción en el contexto latinoamericano, existiendo una plataforma académica y profesional de autores contemporáneos que intentan encontrar una definición local contextualizada de la gentrificación o, al menos, una adaptación de ella en la vida urbana actual.

Específicamente, al focalizar el análisis en el centro histórico de las ciudades latinoamericanas, cuatro situaciones de cambio socio espacial permiten explicar la gentrificación contemporánea: 1) presencia de pobreza urbana y exclusión social dividiendo la ciudad en áreas disímiles(Rodríguez y Winchester, 2004);2) nuevas tendencias en viviendas principalmente localizadas en edificios de vivienda en altura (condominios), vendidas por empresas inmobiliarias y, en la mayoría de los casos, promovidas por los gobiernos nacionales y locales a través de subsidios económicos de adquisición (Hidalgo, 2004; Arriagada et al., 2007; Rojas, 2004); 3) consumidores contemporáneos, caracterizados por personas ligadas a trabajos del sector terciario y de ingresos medios, quienes están eligiendo los barrios centrales para establecer un tipo de vida urbana "de moda" y cerca de sus trabajos (Contreras, 2011; Caldeira, 2000; Arizaga, 2003). Finalmente, 4) esta tendencia de uso está fuertemente ligada con un incremento en el consumo de nuevos departamentos comocommodities, diseñados para jóvenes profesionales, viviendo solos o en pareja, con o sin hijos, e interesados en tener una vivienda con una cierta apariencia global y estándares de seguridad entregados por conjuntos enrejados y cámaras de seguridad (Inzulza, 2012; De Mattos et al., 2005; Jones y Ward, 2004).

Sin embargo, el término gentrificación aun parece ser incipientemente nombrado como tal, manteniéndose como un proceso más reconocido por las clases intelectuales europeas y/o norteamericanas, que las latinoamericanas. En vez del anglicismo gentrificación, términos tales 
como regeneración urbana, renovación urbana (Sargatal, 2000) o mejoramiento de barrio son usados en agendas gubernamentales de nivel nacional y/o local para proponer políticas urbanas, planes reguladores comunales y/o seccionales, o normativas de conservación para mejorar áreas históricas en deterioro y barrios en general (Redfern, 1997). Por lo tanto, es crucial que las tendencias actuales de la gentrificación tengan mayor reconocimiento como un hecho concreto, así como la descripción de sus indicadores, para ser incluidos en las actuales y futuras políticas urbanas propuestas por los gobiernos nacionales y locales.

Específicamente, autores como Jones y Varley (1999), Arriagada et. al. (2007) y De Mattos et. al. (2005) ya han detectado la falta de evidencia empírica de casos concretos para apoyar el análisis de la gentrificación en ciudades latinoamericanas. Más reciente, otros autores como Janoschka y Seguera (2014), Herzer (2008) y Contreras (2011) han ampliado el concepto y contextualizado sus implicancias en ciudades capitales como Buenos Aires y Santiago de Chile. Por su parte, Inzulza (2012) define la "latino gentrificación" como la sustitución de edificación existente de fachada continua o pareada, de uno, dos o tres pisos, de uso principalmente (u originalmente) residencial por una nueva tendencia de residencia con vivienda en media-altura y altura, más que el desplazamiento de clases obreras, o de menores ingresos hacia la periferia de la ciudad por clases de altos ingresos, o el retorno de clases de ingresos medios y altos al centro de la ciudad para establecerse con actividades artístico-culturales.

Es por ello, que la gentrificación necesita nutrirse de casos que permitan cubrir las realidades urbanas contemporáneas y, en particular, cuando en Latinoamérica y el Sur Global en general, existen fenómenos en paralelo que aumentan cambios socio espaciales en centros históricos (Inzulza, 2014). Tal es el caso de los desastres naturales como terremotos y maremotos, los cuales que al igual que la gentrificación, aumentan niveles de pobreza urbana e inequidad social, desplazan residentes y alteran el patrimonio cultural con un evidente cambio en el paisaje urbano (Mitlin y Satterthwaite, 2013; Alexander, 2012).Sin embargo, esta relación de fenómenos parece no estar siendo cubierta masivamente por la academia y menor aun con estudios en ciudades de escala intermedia, la cual representa el $48 \%$ en América Latina (Bolay y Rabinovich, 2004), y muchas de ellas afectadas por desastres naturales como terremotos y maremotos.

Entonces, habría que preguntarse ¿Cómo está respondiendo la planificación urbana la reconstrucción de ciudades latinoamericanas post terremotos? y ¿Qué efectos socio espaciales trae consigo la gentrificación contemporánea en las ciudades intermedias latinoamericanas que están siendo reconstruidas? Además, si acordamos que la ciudad intermedia posee un alto valor de diseño urbano, lo que puede ser reconocido por su escala humana, conformación barrial y relación entre sus residentes que forman grupos conocidos (Poblete, 2013), ¿Existen criterios específicos de diseño urbano que estén siendo incluidos en la recuperación y rehabilitación de barrios históricos dañados por terremotos y/o maremotos en Latinoamérica?

El presente artículo explora los cambios socio-espaciales que está experimentando la ciudad intermedia latinoamericana como resultado de políticas de reconstrucción y gentrificación. Para ello, se adopta una investigación del tipo argumentativo por cuanto refleja una postura crítica con base en los datos recabados, en busca de las causas y de los efectos del proceso de gentrificación en la ciudad de Managua. Además, se pretende dar cuenta de las transformaciones urbanas, sociales y económicas que se han estado dando en el contexto post terremoto del 72 , específicamente, en términos de las escalas: viviendas, asentamientos humanos espontáneos, barrios, colonias y sitios que están estrechamente vinculados con el proceso de gentrificación y con su impacto en la población.

El estudio es empírico y documental, de alcance descriptivo con elementos correlaciónalescomparativos (que permitieran contrastar la realidad de este proceso urbano con lo ocurrido en otras ciudades) y de corte retrospectivo, ya que es una mirada hacia la evolución histórica, 
urbana y social del sitio de estudio. Esto, mediante técnicas e instrumentos que permitieron realizar en un primer momento una investigación documental, y posteriormente la observación y visitas de campo, entrevistas a especialistas en la temática (académicos y funcionarios públicos), entrevistas semiestructuradas, y encuestas por cuestionario a pobladores de la ciudad.

La unidad de análisis es la ciudad de Managua, en un primer momento, su antigua área central (las distintas unidades urbanas de la zona y sus pobladores), delimitada según lo establecido en el Plan Regulador de área central de la ciudad de Managua de 1982, y en un segundo momento el resto de la ciudad que se conoce en la actualidad.

El artículo se estructura en cuatro partes principales. Primeramente, se hace una revisión teóricoconceptual de los procesos e iniciativas de planificación urbana que parecen estar acelerando patrones de cambio socio espacial en el contexto de las estrategias post terremoto adoptadas. Esta dinámica es contrastada con el Diagrama de Ciclo de Alerta del Terremoto (DCAT) el cual permite entender las distintas fases y periodos de tiempo de respuesta dentro de un proceso de rehabilitación urbana post desastre natural.

En segundo lugar, se elige el caso de Managua en Nicaragua, para mostrar empíricamente el proceso de planificación como parte de la reconstrucción post terremoto del 23 diciembre 1972, revisitando cinco hitos historiográficos que permiten dar una radiografía de cómo esta ciudad metropolitana con rol intermedio ha incrementado patrones de cambio socio espacial con marcas de gentrificación contemporánea. Específicamente, se retrata lo sucedido con el evento catastrófico y sus sucesivas etapas de desarrollo y rehabilitación que incluyen implicaciones de políticas sociales de los $1990 \mathrm{~s}$ y el crecimiento del sector inmobiliario, expansión del área metropolitana y nueva política de vivienda en el presente siglo.

Un tercer acápite revisa como los planes "en papel" gubernamentales de planificación de nivel nacional y local fueron aplicados en el centro histórico y resto de Managua, agudizando y acelerando efectos post terremotos que pueden ser asociados con acciones de especulación inmobiliaria, y no necesariamente rescatan los valores implícitos del tejido físico y social que acuñan las ciudades intermedias. Finalmente, se entregan conclusiones que afirman que la gentrificación urbana no es reciente para el caso de Managua, sin embargo, no este proceso es parte del léxico urbano ni de la producción académica local. Es por ello que, tanto el reconocimiento de este proceso, como su vinculación dentro de la reconstrucción, se transforman en una oportunidad para restablecer el ciclo natural de políticas e intervenciones urbanas adecuadas para el crecimiento sustentable de ciudades intermedias.

\section{Cambio socio espacial en las ciudades intermedias}

Aunque el estudio de la gentrificación es similar en la mayoría de la ciudades metropolitanas del mundo, Haase et. al., (2010) plantean que las tendencias contemporáneas de este procesodeberían incluir las ciudades de tamaño medio. En este sentido, las ciudades latinoamericanas son parte de la tendencia de tamaño de rango medio al poseer el48\% de su población viviendo en ciudades de menos de 500.000 habitantes (Bolay y Rabinovich, 2004). Además, parece ser que las ciudades latinoamericanas son más vulnerables que las europeas frente a los efectos de reemplazo que nuevos proyectos de vivienda y edificios corporativos hacen sobre las propiedades residenciales afectas a la reconstrucción post-desastres (Onestini, 2011). Wamsler (2007) reconoce que desde "el periodo de la colonia, una planificación deficiente promovió fuertemente la vulnerabilidad de las ciudades latinoamericanas [las cuales] ...comúnmente fueron localizadas por razones de acceso económico y producción más que factores de seguridad, transformándolas en casos de riesgo" (p.25). 
Esta situación puede ser asociada además con los orígenes de la urbanización en Latinoamérica (United Nations, 2012), cuando la gran mayoría de las grandes ciudades [allí fueron] asentadas en lugares inadecuados [riesgosos], ya sea en faldas de volcanes, en suelos saturados de agua, en las márgenes de ríos, franjas costeras o fallas sísmicas activas... y, estas condiciones de riesgo se han venido agudizando por el incremento acelerado de elementos expuestos (físicos y humanos), pero también por una radical transformación del territorio" (Mansilla, 2011:20). De esta forma, los procesos de reconstrucción abren el debate sobre como las tipologías de gentrificación contemporáneas están siendo aplicadas para rehabilitar las áreas centrales promovidas con sociedades público-privadas en nombre de la regeneración urbana y mejoramiento de ciudades.

En ese sentido, los centros históricos son vistos como "objetos del deseo" (Carrión, 2005) para promover proyectos de vivienda y comercio de moda, logrando capturar las necesidades de las clases medias latinoamericanas. Es por ello que la gentrificación contemporánea retratada como el redesarrollo de sitios eriazos (brownfield) por Davidson and Lees (2010) en Inglaterra, se puede relacionar con la situación de los centros de ciudades afectados por terremotos y maremotos, los cuales presentan gran cantidad de sitios vacíos a la espera de nuevo desarrollo inmobiliario. Dentro de este contexto, se puede reconocer como 'heridas' sociales al riesgo de desplazamiento de residentes existentes, especialmente cuando negocios 'ilegales' son llevados a cabo dentro de las primeras etapas de reconstrucción.

De este modo, las buenas condiciones que poseen las ciudades intermedias como la escala humana, encuentro de grupos conocidos, memoria colectiva (Hayden, 1995), paseabilidad e identidad barrial (Lynch, 1960), parecen estar modificándose rápidamente por tipologías de vivienda nueva y otros usos como comercio y oficina que utilizan los centros históricos y bordes costeros. Proyectos de altura media y altura localizados en una ciudad con una adecuada dimensión física permite a los nuevos residentes acceder peatonalmente sin demasiada dificultad, a los principales servicios y equipamientos de la ciudad o desplazarse, sin demasiado esfuerzo, a cualquier punto del espacio urbano (Bellet y Llop 2002:254). Dentro de las causalidades, se pueden nombrar a fenómenos como la segregación urbana con sus brechas de desigualdad, así como la carencia de políticas de desarrollo local en el sentido de incentivar integración y diversidad.

Sin embargo, parece haber poca investigación que permita identificar el rango de condiciones tales como localizaciones, estructura de tenencia del suelo y acuerdos de gestión que los proyectos de edificación en altura deberían incluir antes de ser localizados en centro de ciudades intermedias para evitar mucho de los problemas que son encontrados...en otras localidades [como] la 'regeneración urbana' en alta densidad (Kearnset al., 2012:19). Sin duda que la transformación de ciudades con un carácter global como lo es la gentrificación contemporánea, trae como consecuencia la estandarización de estilos de vida, culturas de conductas y formas, a menos que exista una relación consciente entre arquitectura y paisaje, y como los planes maestros podrían incluir estudios de la altura de los objetos y proteger vistas de gran valor de bellezas naturales (Lukić, 2011:131).

\subsection{Aceleración en el contexto post terremoto}

Olshansky and Chang (2009) enfatizan que "a pesar de nuestros esfuerzos, los desastres naturales seguirán ocurriendo, y los planificadores urbanos juegan un rol crucial en el sentido de guiar la recuperación de lo que ha sido destruido" (p.200). Además de las necesidades básicas de "alojamiento permanente, empleo, suministro de cuidados de la salud y educación, etc., [las personas afectadas tienen] también necesidades sociales, culturales y psicológicas que incluyen problemas de identidad que significan sentido de formulación y mecanismo de predicción"(OliverSmith, 1994:11). En este sentido, las estrategias post-desastres deberían ayudar a reconstruir 
el tejido físico y social que posee un barrio, una vecindad u otra unidad identificable en la ciudad. La gente y sus medioambientes están íntimamente unidos, entonces, los procesos de reconstrucción deberían incluir además "elementos culturales e imágenes relevantes para la comunidad afectada tales como iglesias, capillas, santuarios, plazas, barrios y escuelas, pero además algunos simbólicos elementos como árboles lugares de reuniones informales" (OliverSmith, 1994:12).

Sin embargo, una diferente realidad es escrita por investigadores centrados en estrategias de reconstrucción. La mayoría coinciden que desde los 1990s los procesos de reconstrucción están siendo vistos como una oportunidad para invertir en proyectos nuevos bajo la fuerza del Mercado y con especulación inmobiliaria, más que la implementación de estrategias para enfrentar adecuadamente los efectos físicos, sociales y psicológicos (Ozerdem y Rufini, 2013; Mansilla, 2011; Lawner, 2010; CEPAL 2010). En general, parece no existir una conciencia del "riesgo de desastre en la planificación urbana ni regional, [ni en] la regulación del crecimiento urbano (PNUD, 2004:88). Wamsler (2007) ratifica esta aserción señalando que "publicaciones que incluyan la adaptación de planificación para el desarrollo de asentamientos que están sujetos a desastre son una excepción... [y] principalmente son enfocados en los aspectos puramente físicos/estructurales del medioambiente construido formalmente en países desarrollados" (p.26).

De esta forma, la reconstrucción post-terremoto puede entenderse erróneamente (o únicamente) como una respuesta rápida al daño físico con provisión de vivienda y recuperación de servicios básicos (suministro de agua, electricidad, conectividad primaria, etc.). "Esto a menudo significa el no considerar las condiciones pre-existentes de riesgo de desastre y de este modo ignorar efectivamente el riesgo de futuros desastres" (IRP, 2007:2). Por ejemplo, experiencias recientes de reconstrucción como el terremoto M6.3 de L'Aquila, Italia en 2009 ha significado principalmente 'la politización' de este proceso y además un falta de coordinación y de tenencia local en todos los niveles (nacional, regional y municipal), incluyendo incluso ciertos derechos conferidos y corrupción (Ozerdem y Rufini, 2013).

Sólo algunos casos han sido focalizados en las consecuencias socio-económicas, culturales y emocionales de las comunidades afectadas, en especial de los residentes originarios, incorporando marcos de acción de planificación estratégica a corto, mediano y largo plazo para confrontar terremotos (PNUD, 2004). Y aún menos investigación parece focalizarse en evaluación de riesgo, actitud cívica y estrategias de resiliencia de la comunidad (Moseret al., 2011). Dentro de este contexto, algunas buenas prácticas tales como el reporte de la República de Indonesia (2005) y el 'olvidado' plan maestro de reconstrucción de Skopje (Lozanovksa, 2012) muestran como los gobiernos nacionales y locales pueden incluir además la reconstrucción de la comunidad, su economía de escala, infraestructura y formas de gobernanza apropiadas.

Para el contexto de América Latina y El Caribe, una institución clave es El Centro Regional de Información sobre Desastres (CRID), el cual se constituye como una iniciativa patrocinada por seis organizaciones ${ }^{1}$ que decidieron unir sus esfuerzos para asegurar la compilación y entrega de información sobre desastres para este continente. Esta iniciativa ha contribuido, sin duda, con el análisis y registro de terremotos para esta Región y, más exhaustivamente, con la evaluación de políticas urbanas de reconstrucción (WeissFagen, 2008). Sin embargo, Mitlin and Satterthwaite (2013) nos recuerdan que la nueva pobreza es uno de los indicadores cruciales en el Sur Global

${ }^{1}$ Estas organizaciones son: (1) Organización Panamericana de la Salud - Oficina Regional de la Organización Mundial de la Salud (OPS/OMS); (2) Naciones Unidas, secretaría de la Estrategia Internacional para la Reducción de Desastres. (ONU/EIRD); (3) Comisión Nacional de Prevención de Riesgos y Atención de Emergencias de Costa Rica (CNE); (4) Federación Internacional de Sociedades Nacionales de la Cruz Roja y Media Luna Roja (FICR); (5) Centro de Coordinación para la Prevención de los Desastres Naturales en América Central (CEPREDENAC) and (6) Oficina Regional de Emergencias de Médicos sin Fronteras (MSF). 
y además encontrado como efecto directo e indirecto de los terremotos; pero pese a ello, este factor ha sido desestimado en la mayoría de los reportes que analizan los procesos de reconstrucción.

\subsection{Diagrama de Ciclo de Alerta del Terremoto (DCAT)}

Los terremotos significan de alguna forma un proceso permanente, considerando la frecuencia con que se registran en general estos desastres naturales en países que son parte del 'Cinturón de Fuego'2 (USGS, 2013; SVS, 2012). Si se considera que un terremoto de gran magnitud es registrado cada década en algún punto del territorio latinoamericano como promedio (ver sitio web CRID), el objetivo de incluir este tipo de desastre natural dentro de un marco de acción de planificación regular parece ser obvio. Sin embargo, la realidad de los procesos de recuperación post-terremoto nos dicen que los problemas para enfrentarlos se repiten en la mayoría de estos casos.

Especialmente, cuando se revisan los diferentes enfoques para implementar estrategias a través de un periodo de tiempo "donde 'lo temporal' a menudo se transforma o se extiende a 'décadas', y donde lo 'transicional' se deja finalmente como una modificación permanente" (Ozerdem and Rufini, 2013:136). Por lo tanto, es crucial entender que los terremotos son parte de un ciclo natural que requieren de un enfoque estratégico y original para enfrentarlos, y con la inclusión de planes urbanos estratégicos que incluyan las diferentes etapas de acción y períodos de tiempo (Caldera Sánchez, 2012). Este planteamiento podría además entenderse como un enfoque más sustentable que otras acciones, las cuales muchas veces, son propuestas como estrategias postterremotos con nueva visión estética para mejorar ciudades dañadas, y no necesariamente incluyendo el tejido socio espacial originario de los barrios afectos.

La Figura 1 muestra el "Diagrama de Ciclo de Alerta del Terremoto (DCAT)", el cual incluye las principales etapas o fases y los periodos de tiempo que son parte de un marco de acción estratégico de reconstrucción. Para ello, es fundamental entender que cada ciudad y pueblo necesita adaptar su propio 'reloj despertador' como ciclo de alarma para enfrentar este riesgo natural considerando que un terremoto pone en evidencia lo mejor y lo peor de un sistema de planificación territorial. En ese sentido, los terremotos son de alguna forma eventos naturales posibles de predecir si se analizan dentro de una base de datos y registros sistematizados, como por ejemplo, lo llevado adelante por el Centro Regional de Información sobre Desastres (CRID). Entonces, si acordamos que los terremotos son parte de un "ciclo natural" (Olshansky and Chang, 2009), es posible planificar adecuadamente el desarrollo de ciudades incluyendo sus fases de acciones y dentro de períodos de tiempo acordes a cada fase.

${ }^{2}$ Además llamado 'Circum-PacificBelt' incluye los siguientes países: Chile, Peru, Ecuador, Colombia, Centro América, México, Estados Unidos, Canada, Islas Aleutianas e islas de Rusia, China, Japón, Taiwan, Filipinas, Indonesia, Papua Nueva Guinea, Australia y Nueva Zelanda. 


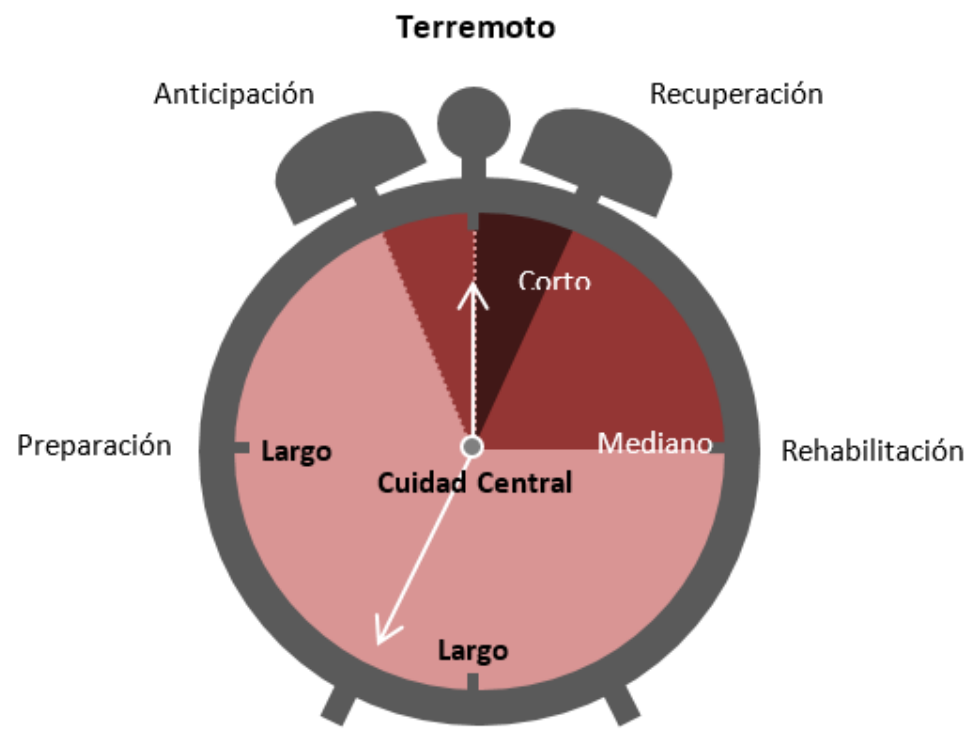

Reconstrucción

Fig. 1 Diagrama de Ciclo de Alerta del Terremoto (DCAT)

Fte: Inzulza, 2014

Una vez que ocurre un terremoto, lo cual significa segundos o pocos minutos, un proceso sin retorno se activa como un cronómetro o temporizador. De acuerdo con autores expertos en el tema, es posible acordar cuatro fases principales que le siguen a este evento natural, que son Recuperación, Rehabilitación, Reconstrucción y Preparación (Ozerdem y Rufini, 2013; PNDU 2010; CEPAL, 2010; Wamsler, 2007). Además, este diagrama DCAT es propuesto para las áreas centrales de ciudades que normalmente poseen un patrimonio arquitectónico y cultural valioso. Específicamente, las áreas centrales con sus barrios y residentes originarios constituyen el espacio físico y social más importante de ciudades intermedias, el cual normalmente presenta daños importantes por causa de terremotos y maremotos. Pero de la misma forma, el regreso al centro como fenómeno global y actual observado por David son and Lees (2010) es parte de ciudades de escala intermedia, y entonces fundamental de incorporar, en particular, con el comportamiento de los nuevos residentes que están implícitos dentro del proceso de la gentrificación contemporánea.

En síntesis, a partir de este diagrama conceptual, es posible, por una parte, entender que los aspectos sociales, económicos y políticos son, de alguna forma, determinantes en la estructura urbana de ciudades intermedias, siempre y cuando se entiendan dentro de un proceso continuo y cronometrado y, por otra parte, que la gentrificación y los desastres naturales parecen estar actuando cada vez más de la mano, entonces requieren ser entendidos como problemáticas y contrarrestados a través de fases de acción de corto, mediano y largo plazo. Esta doble correlación es posible de observar en el caso de estudio que se presenta a continuación, el cual es organizado en cinco hitos historiográficos principales.

\section{Managua: una ciudad metropolitana con rol intermedio}

Managua es la ciudad capital de Nicaragua (ver Figura 2) desde 1857y posee un extensión territorial de $6,197 \mathrm{Km}^{2}$, con una población de 966,518 habitantes (INIDE, 2005), lo cual corresponde al $\mathbf{1 7 . 6 8} \%$ de la población total del país. Esta es la ciudad de mayor densidad poblacional de Nicaragua, y forma parte de la Región Metropolitana del mismo nombre, junto a 
otros 29 municipios (ver Figura 3). A lo largo de su historia, es posible reunir varias condicionantes antrópicas que han influido en la conformación del espacio, por lo que el aspecto natural es determinante para poder entender la ciudad.

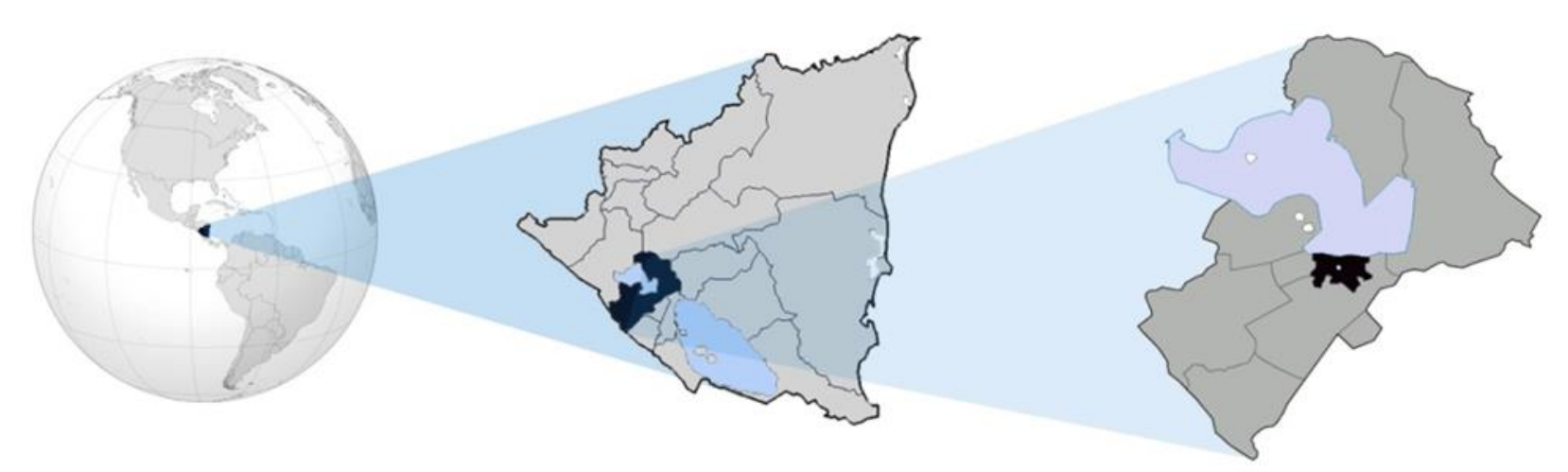

centro américa, américa managua, Nicaragua

MUNICIPIO Y CIUDAD

DE MANAGUA

Fig. 2 Macro y micro localización de la ciudad de Managua Fte: López y Suarez, 2013

Antes del terremoto del 72, la ciudad de Managua conto con dos instrumentos que guiaron su crecimiento, estos fueron el Plan Regulador de Managua de 1954 y 1968. En este periodo de 1954 a 1972, los planes reguladores fueron los únicos instrumentos legales para controlar y orientar los usos de suelo y la construcción en la ciudad de Managua, pero dejando poco claro los lineamientos para expansión de la ciudad. Estos instrumentos fueron concebidos como Planes Reguladores del Desarrollo Urbano de la ciudad de Managua, donde la zonificación básica de los usos de suelo era su principal función. En términos generales los planes carecían de estrategias urbanas integrales, pero por la escala de la ciudad en esos años, estos instrumentos satisfacían las necesidades generales de la ciudad y proporcionaban algunas directrices que permitían tener cierto control urbano de la capital, que además era una ciudad compacta y con una retícula ortogonal bien definida.

Por otra parte, la revisión de los desastres a causa de fenómenos naturales, no solo permite analizar la historia y la información sobre el tejido urbano de la ciudad, sino que además, entregaelementos de análisis cruciales acerca del cambio socio espacial contemporáneo que se ha dado en ella. Hardy (2000) alude al respecto:

"A raíz de los distintos fenómenos naturales que han afectado a la ciudad de Managua, la población con mayores recursos económicos empezó a distribuirse en islotes urbanos acondicionados por las autoridades locales, con el fin de no quedar expuestos a los riesgos naturales. La estructuración de la sociedad capitalina favoreció esta política. La creación de zonas urbanas dotadas de todos los servicios, contrasta con otras zonas que carecen de condiciones mínimas de habitabilidad. Como resultado de esto los sectores sociales de Managua se fueron desarrollando de forma desigual, dando lugar al aparente surgimiento de dos ciudades en vez de una sola. De esta forma se evidencia el deterioro de las condiciones de habitabilidad de los pobladores en las zonas más precarias de la ciudad, lo que ha consolidado un proceso de exclusión social y segregación espacial." (p.85). 


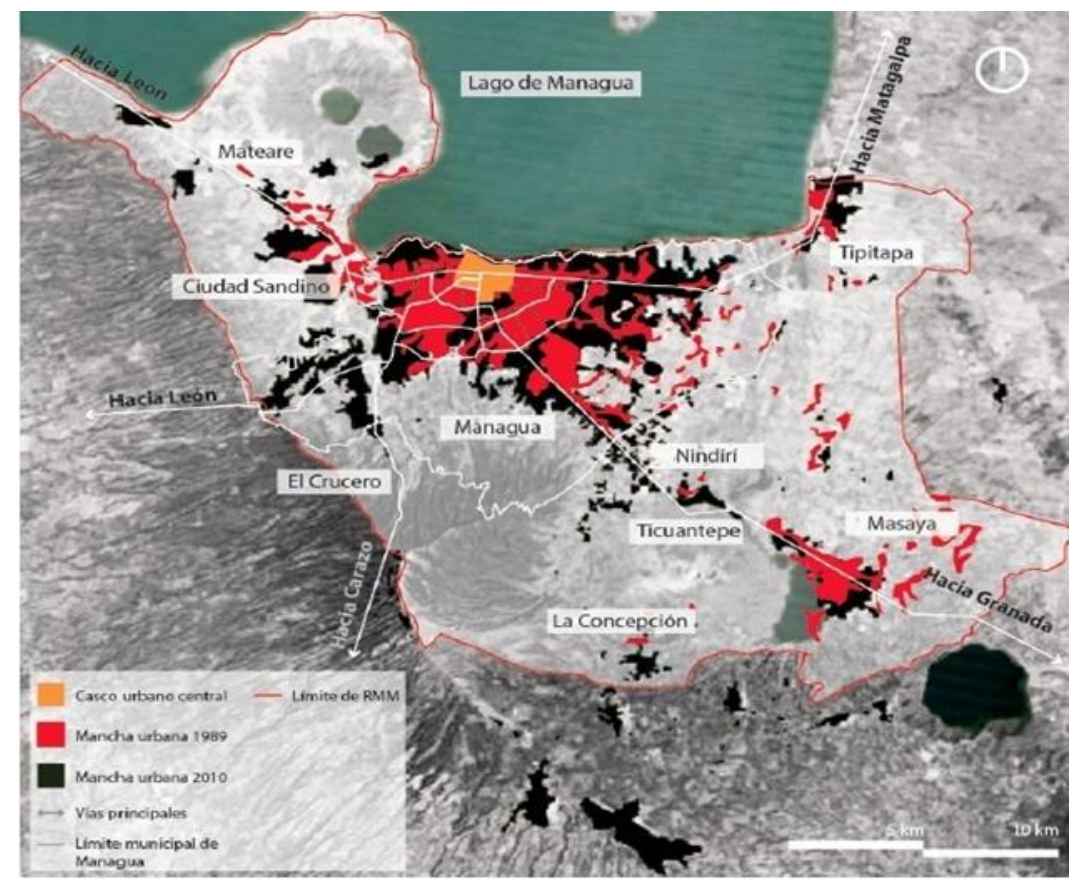

Fig. 3 Región Metropolitana de Managua a 2010

Fte: BID, 2014. Plan de acción: Managua sostenible-Iniciativa ciudades emergentes y sostenibles

\subsection{El terremoto del 23 diciembre 1972: inicio de la transformación socio espacial}

Con el sismo de diciembre de 1972 se desintegra la estructura urbana de damero de la ciudad de Managua, y se impide la reconstrucción de su centro por recomendaciones de un estudio realizado después de la catástrofe, lo que fomentó la dispersión de la población para evitar la concentración de daños en caso de otro desastre. Con esta política urbana pionera, se inicia una de las mayores especulaciones de las propiedades inmuebles. La familia Somoza ${ }^{3}$ contaba con todos los recursos para comprar las tierras ubicadas en la periferia de la ciudad, y controlar por completo la reconstrucción que se realizó en los terrenos ubicados alrededor del centro histórico y de los ejes viales más importantes.

La creación de nuevos espacios residenciales era una prioridad. Se sabía dónde se debía construir (fuera del centro histórico), sin embargo no se definió una estrategia de crecimiento integral, lo que provocó un sin número de predios vacíos entre espacios construidos. Había una población originaria que se quedó en los sitios, otra que migró a otras partes de la ciudad o fuera de ella, y población de otros sitios del país que vio la posibilidad de asentarse y establecerse en la capital, sobre todo en los barrios costeros. La población de escasos recursos económicos hace uso de la zona de desastre, pero en una condición de ocupación ilegal de los edificios en ruinas, lo que se convirtió en un argumento sólido para desligarse de responsabilidades por parte de las autoridades sobre la dotación de bienes y servicios a estas zonas.

"Como el resultado del desplazamiento de los residentes acomodados que anteriormente habitaban en el destruido centro de la ciudad, las tierras aledañas a las carreteras fueron las principales en ser desarrolladas. Este brusco proceso de

\footnotetext{
${ }^{3}$ La familia Somoza, gobernó Nicaragua por más de 40 años bajo una dictadura militar, hasta que el 19 de julio de 1979 fue derrocada por el Frente Sandinista de Liberación Nacional FSLI.
} 
urbanización sin ninguna regulación urbana, coincidió con la proliferación de nuevas vías de tránsito vehicular en la periferia urbana." (EDUM, 1982:30).

Los cambios en el centro histórico y en el resto de la ciudad no sólo provocaron afectaciones físicas con la división espontánea, re-poblamiento y despoblamiento de algunas áreas, sino también conllevó a la re-significación del espacio en una ciudad que ya no era la misma, y con sus relaciones y convivencia fuertemente afectadas.

\subsection{Periodo 1973-1978: política urbana en el contexto de estrategias de post-terremoto}

Posterior al terremoto de 1972 el crecimiento de la ciudad estuvo determinado por la reconstrucción, desarrollándose funcionalmente hacia el este y al sur en menor grado. Se dio la transformación de usos de suelo de vivienda a comercio como es el caso de Ciudad Jardín, y se construyeron en la zona periférica centros comerciales como el Centro Comercial Managua. Las áreas de gobierno y administración se concentraron en un conjunto de edificaciones diseñadas y construidas para viviendas (Centro Cívico, ubicación actual de las oficinas de la Alcaldía Municipal). Las instalaciones militares se consolidaron en la Loma de Tiscapa y las oficinas de carácter político (Gobierno) se relocalizaron en áreas verdes y comunales.

Todos estos cambios fueron haciendo que en algunos sectores habitacionales perdieran su identidad, y la posibilidad de que las familias permanecieran en sitios cercanos a centros de educación y servicio, generándose un mayor desplazamiento de grupos. Mientras tanto el área central de Managua seguía en total abandono por parte de la administración pública y cada vez era más la gente de escasos recursos que ocupaba parte de los predios vacíos y edificaciones en ruinas.

En 1978, seis años después del terremoto, todavía perduraba entre la población, la idea de que aún no se había iniciado el proceso de reconstrucción del centro histórico de la ciudad, el cual estaba ahí sin ninguna función. El geógrafo Michel Foucher, publicó en 1980 un artículo que tituló "Managua ciudad atomizada", y utilizó la expresión "ciudad en pedazos" para describir este territorio urbano tan inusual. El término de ciudad atomizada remite a la idea de una sociedad dual, desigual, fragmentada y segregada, y el término de ciudad en pedazos por su parte, invita a pensar sobre una pérdida por cortes y rupturas de la unidad conceptual de la ciudad. El tejido urbano destruido fue sustituido por numerosos espacios discontinuos (Bolonia, la Centroamérica, barrio Larga Espada, Bello Horizonte, Parte de Villa Fontana), que se transformaban continuamente mientras en centro histórico de la ciudad las condiciones de abandono seguían (ver Fig. 4).

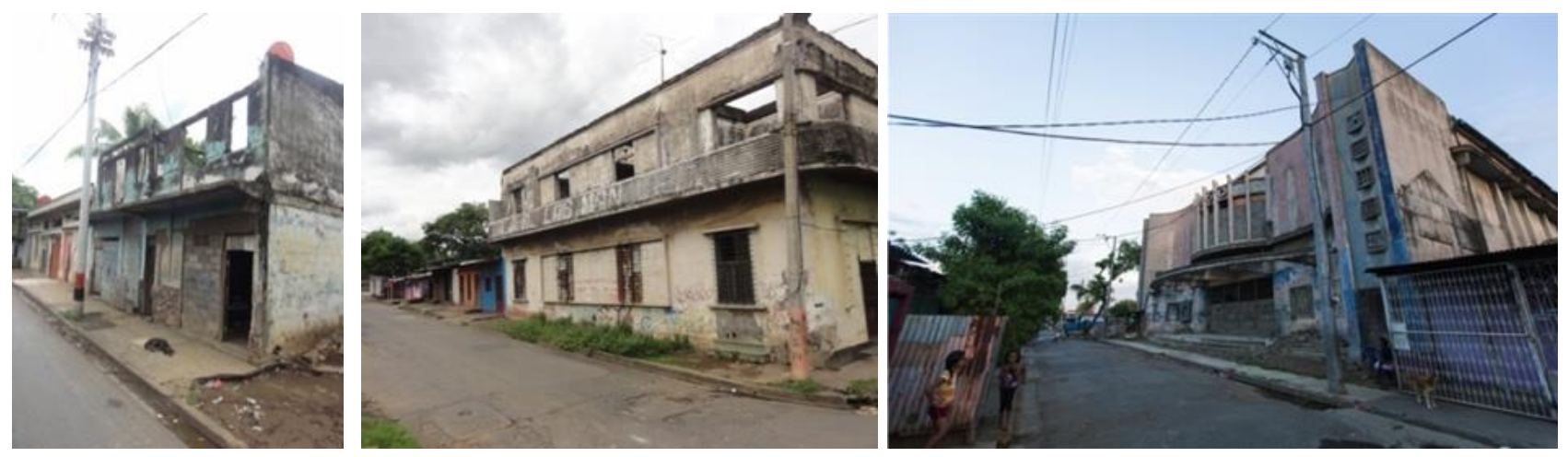

Fig. 4 Centro histórico de Managua: barrio La Candelaria en situación de abandono

Fte: Saravia, 2013 y BID, 2014. Plan de acción: Managua sostenible - Iniciativa ciudades emergentes y sostenibles. 
De esta forma, en esta primera etapa pos-terremoto manifiestan características propias de los procesos de gentrificación, ya que se parte de la intervención del estado sobre zonas urbanas para favorecer la especulación del sector inmobiliario o intereses económicos, que buscan la transformación urbana de un sector, la exclusión social de la población y el cambio en la tipología arquitectónica de las áreas afectadas, en este caso, la zona central de la ciudad de Managua y su entorno inmediato.

\subsection{Periodo 1979-1990: implicaciones de políticas sociales}

El entender el espacio social desde el enfoque político es también importante, ya que define a este ámbito como un objeto de la política y la toma de decisiones. Así por ejemplo, Camarena (1987), encuentra en ello otras condiciones determinantes en los procesos urbanos, sobre esto el menciona:

"La correspondencia entre espacio social y organización política y social puede presentar distintos niveles y grados de articulación y coherencia, pero invariablemente su relación está sujeta al tipo de uso, transformación, reorganización, de acuerdo con el cambio en el objetivo para el cual fueron ideados y experimentados" (Camarena, 1987:67).

Bajo la premisa mencionada anteriormente es comprensible entender que tras el fenómeno natural y la modesta reconstrucción del centro histórico de la ciudad, otro suceso que condiciona la articulación espacial de la ciudad es el triunfo de la Revolución Popular Sandinista del 19 de julio de 1979. Tras perseguir el derrocamiento de la dictadura somocista, el nuevo gobierno revolucionario hereda una ciudad anárquica, sin servicios ni equipamiento adecuado a las necesidades de la población. La prioridad es la unidad y reconstrucción nacional, con una clara tendencia al ordenamiento territorial descentralizado.

Para ese período, en Managua se dan obras encaminadas a la recuperación del centro histórico de la ciudad y la reconstrucción de los edificios para la ubicación de actividades administrativas, construcción de vías (Dupla Norte y Sur), áreas recreativas como el parque Luis Alfonso Velásquez, construcción de viviendas y proyectos de gran importancia para el desarrollo de la ciudad como son los intentos por recuperar el Lago Xolotlán, y solucionar el problema del drenaje pluvial. Por otra parte, en 1983, la Alcaldía de Managua (ALMA) y el Ministerio de la Vivienda y Asentamientos Humanos (MINVAH), operativizan parte del Plan Regulador de Managua, creado en 1982, el cual contempla como estrategia urbana del plan la saturación del área urbanizada para optimizar el uso de suelo de las inversiones existentes en infraestructura, equipamiento y servicios urbanos.

"El cambio que se dio a nivel urbano en los 80 fue muy poco, esto de hecho obedeció, en primer lugar, a que los habitantes de Managua y el resto del país debieron enfrentar el bloque económico impuesto por E.E.U.U. y a la guerra de la contrarrevolución." (Hardy, 2000:77)

En tal contexto, las estrategias urbanas que el gobierno Sandinista llevo a cabo durante los años ochenta, tan solo produjeron la efímera ilusión de que se estaba creando vínculos entre los habitantes y los espacios urbanos de la capital. Tales políticas no tomaron en cuenta el hecho de que el espacio urbano de Managua no estaba regido por un mero proceso de diferenciación socioespacial, sino también por un complejo proceso de fragmentación urbana. Lo anterior no se limita a describir la realidad urbana de Managua, de sus mutaciones y transformaciones físicas y de los comportamientos sociales de los ciudadanos, también explica la aparición de una nueva forma urbana, característica de los procesos de gentrificación que se estaba dando en la ciudad. Sin embargo, este proceso no se daba propiamente en el centro histórico de ésta, el cual continuaba en abandono, y donde las pocas intervenciones daban indicios que el camino que se estaba gestando también era parte del cambio socio espacial, por tres aspectos básicos como lo eran: 
el cambio de uso de suelo, de tipología arquitectónica y, el más evidente, el cambio de los pobladores de la zona que se inicia desde el terremoto de1972.

"La ciudad se transformó en un espacio amplio con núcleos poblacionales dispersos, siendo las zonas orientales las más densamente pobladas. Hacia 1983 más del 60.7\% de los pobladores de este sector de la ciudad vivían en 7 barrios creados después del terremoto: Las Américas I, Villa Revolución, Villa Venezuela, Villa Flor, Villa Libertad, Jardines de Veracruz y Rubenia. La afluencia de la población hacia estos barrios deficientes en infraestructura y servicio, contribuyó a convertirlos en menos de diez años en lugares no solamente repulsivos sino también expulsivos" (Barahona, 1985:12).

De esta manera, se fue perfilando nítidamente un cambio con respecto al uso de suelo urbano de la ciudad. Las zonas que anteriormente se ubicaban en la periferia de la ciudad, se fueron integrando al tejido urbano, sin que este resultara saturado, quedando grandes espacios vacíos en el centro y otras zonas de la ciudad.

\subsection{Periodo 1991-2006: crecimiento del sector inmobiliario}

El inicio del1990 marcó el regreso de los liberales al poder, y con ello una restructuración en la política estatal. El desarrollo urbano de la ciudad siguió realizándose fuera de su antiguo centro histórico, y la invasión de tierras siguió evolucionando, ahora más en las zonas periférica, así como espacios en situaciones de riesgo a orillas del lago y causes. Por otra parte, los nicaragüenses que se encontraban en el extranjero, inician la gestión legal para recuperar sus antiguas propiedades, abandonadas durante los '80. Estos pobladores recién llegados, también demandaron mejores viviendas, y muchos de los ciudadanos comenzaron a ver como una ventaja el reubicarse a espacios más seguros que los que habían dejado. Se vuelve un peligro el habitar cercano a un asentamiento, en unas viviendas que además ya no cumplen con sus expectativas, lo que aumenta la demanda de los sectores residenciales conocidos (iniciados aproximadamente en 1995).

En el antiguo centro histórico de la ciudad de Managua se da la reconstrucción del antiguo Gran Hotel y Palacio Nacional. Al mismo tiempo, se observó deterioro de zonas habitacionales populares impulsadas por el gobierno anterior (San Antonio y San Sebastián, las llamadas palomeras) que tuvieron como objetivo la revalorización del suelo y el aprovechamiento de la infraestructura de la ciudad. Además, se construyeron nuevos y costosos hitos urbanos tendientes a modificar la estructura simbólica del sitio: la fuente luminosa en el área de la plaza central de la antigua catedral, la rotonda de plaza inter y del malecón. Todos estos aspectos siguieron aportando al proceso de gentrificación por la fuerte transformación que causaron en el paisaje urbano de la zona.

\subsection{Periodo 2007-2014: expansión del área metropolitana y nueva política de vivienda}

En estos últimos años, el ordenamiento de la ciudad se ha tratado de realizar con los Planes Parciales de Ordenamiento Urbano de los sectores Occidental, Oriental y Central (2006). Estos contemplan una caracterización general de la ciudad y propuestas de mejora y desarrollo para los distintos sectores urbanos. Sin embargo, la misma concepción de planificar dividiendo la ciudad, ha inducido a una falta de articulación de la realidad urbana y sus pobladores, lo que deja en evidencia la falta de gestión urbana para la ciudad. En los períodos anteriormente mencionados se consolidan los ejes de crecimiento de la ciudad y se trata de dar el repoblamiento de las zonas baldías. Sin embargo, la mayoría de los asentamientos humanos del área central y del resto de la ciudad se encuentran en situación de ilegalidad y riesgo. Además son poblaciones en condiciones económicas y sociales muy parecidas, totalmente desprovistas de infraestructura y servicio. 
En el 2008, se inicia el proyecto habitacional "Casas para el Pueblo" ejecutado por la alcaldía de Managua y el gobierno central, el cual comprende una serie de casas distribuidas en todos los espacios baldíos de la antigua área central de la ciudad. Este tipo de proyectos en sus inicios buscó consolidar estas áreas. Sin embargo, esta intervención no logra integrar a los barrios existentes, al no desarrollar espacios públicos comunes que articulen las viviendas distintas a las del sitio y con carencia de un plan de reconstrucción vial. Además, los pobladores a los que se les asigna esta vivienda, son foráneos en su mayoría, lo que genera cambio en la población del sitio. De esta forma, los patrones mencionados son propios de los procesos de gentrificación donde se dan cambios de usos de suelo, variantes en la tipología urbana-arquitectónica de las nuevas intervenciones con relación a la existente, y una exclusión social con desplazamiento de la población de la zona (ver Fig. 5).
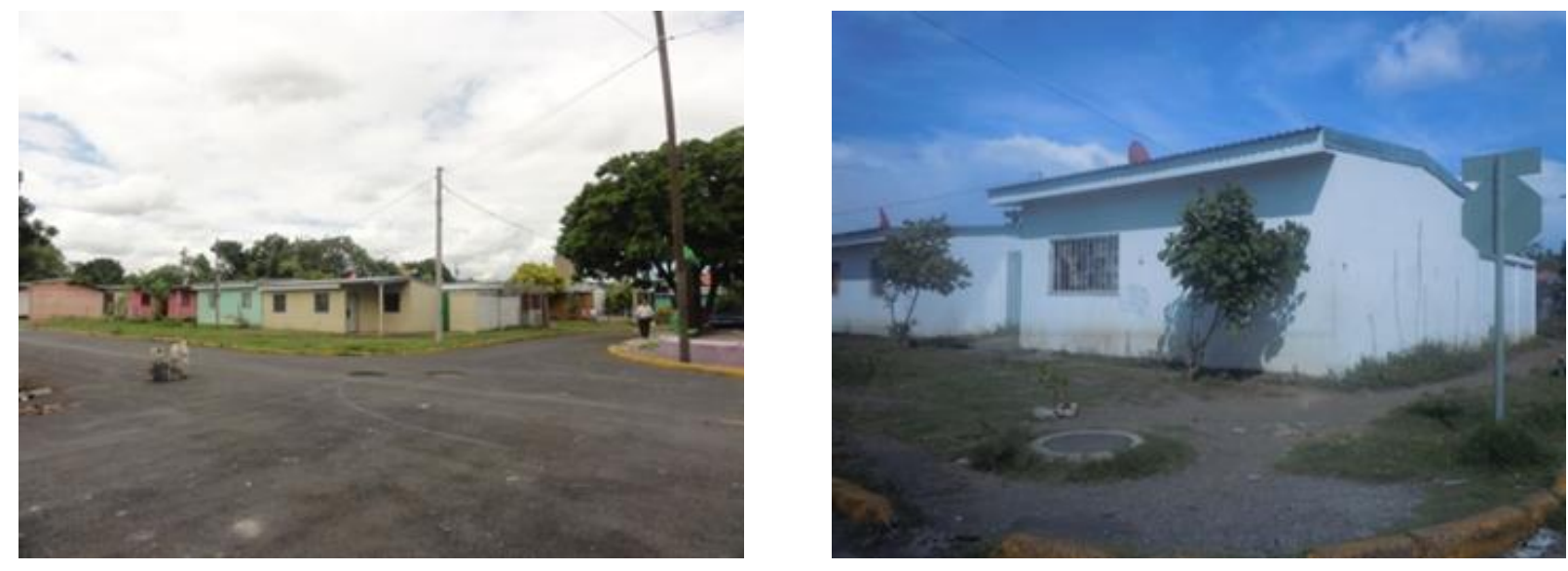

Fig. 5 Casas para el Pueblo

Fte: Saravia, 2013

Por otra parte, la ciudad de Managua se convierte en una ciudad de sub-centros, donde los centros comerciales, colegios y empresas son el eje que la estructura. La ciudad favorece ampliamente el proceso de atomización privatizante que genera distintos tipos de gentrificación. El más claro se da en los últimos años con la "peri-gentrificación", cuyo proceso ha sido documentado para otras ciudades como Metepec, México (Becerril-Sánchez et al., 2013). Otro de los aspectos que contribuyen a estos procesos de gentrificación en Managua es la carencia en la administración pública, ya que el gobierno local no administra totalmente el territorio urbano para el conjunto de habitantes, dándose una desvinculación en las intervenciones urbanas de la ciudad. Además, se suma la ausencia de planes urbanos integrados y sostenibles que permitan la construcción de una ciudad inclusiva y compacta.

Finalmente, en esta época Managua se consolida como una ciudad de servicio, donde prima la rentabilidad del suelo, dando paso a la gentrificación de espacios residenciales dentro de la ciudad, y con ello, aportando mucho más en cuanto a recaudación se refiere. Además, el área metropolitana crece desmedidamente con la diversidad y división de los espacios en una ciudad que cada día es más extensa, y con claros indicios de estratificación. Lo anterior complejiza aun más la realidad, ya que la mayoría de las urbanizadoras, en la zona de carretera a Masaya y León, ya no pertenecen a la ciudad, y por tanto, están ocasionando afectaciones a otros municipios como Nindirí y Ticuantepe, donde las poblaciones originarias también están siendo desplazadas y poco a poco repiten el mismo modelo urbano de la ciudad de Managua. 


\section{Los "planes en papel" frente al proceso de gentrificación en Managua}

Como se revisó en las secciones precedentes, el proceso de gentrificación urbana no es reciente, y a lo largo del tiempo ha venido modificando su definición y sus tipos según las características propias de cada época y según las tendencias de evolución de las ciudades. Si se trata de concertar la posición del Estado o la Municipalidad de Managua frente al proceso de gentrificación, nos encontramos que no ha habido un planteamiento específico de este por no decir, una carencia de conocimiento sobre esta temática y sus repercusiones directas en la ciudad. Han existido planes urbanos con una fuerte inclinación a lo espacial, dejando a un lado la perspectiva social. Esto mismo ha sucedido con las políticas sociales, mayormente expresadas en el mejoramiento de barrios y construcción de nuevas viviendas, donde se desarticulan los distintos elementos del espectro urbano, y sus manifestaciones favoreciendo al desarrollo de este proceso.

Afirmar que la gentrificación es producto de una mala gestión urbana de la administración pública, resultaría inapropiado, debiendo reconocer que este proceso no posee un único aspecto que lo genera.Sin embargo, los cinco hitos historiográficosanalizados, nos permiten detectar acciones que se han ido vinculandocon este proceso de cambio socio espacial a través del tiempo. En ese sentido, lo más gravitante parece ser las acciones de planificación que se han dejado de hacer para evitar el desarrollo de un modelo de ciudad dispersa y fragmentadaque no favorece la convivencia de los habitantes. Esto se manifiesta muy claramente al ver a Managua con un constante crecimiento desordenado y difuso, sin una lógica unificadora como ciudad, donde existe una carencia de políticas integrales de desarrollo urbano sostenibles de acuerdo a las exigencias que presenta la ciudad.

Ello queda demostrado aún más cuando desde el 1982 a la fecha, se pueden contabilizar más de 15 instrumentos relacionados con la planificación de la ciudad, los cuales no han logrado su conexión, coherencia o articulación entre ellos. De esta firma, la gestión y planificación urbana en el caso de Managua, no ha sido un instrumento veraz ni ha sido un recurso beligerante, más bien, ha estado en un bajo perfil y muy permisible en relación a este proceso de gentrificación. En ese sentido, la gestión urbana ha dejado que sean otros agentes los que buscan el crecimiento de la ciudad y ha permitido además que se aumente el costo de las inversiones públicas. La alcaldía parece valorizar el suelo, a través del incentivo al desarrollo inmobiliario y pierde mucha recaudación porque no posee instrumentos que hagan robustos los planes existentes.

De hecho, uno de los argumentos que se han utilizado para promover el desarrollo de residenciales cerrados en la ciudad de Managua, es que mediante esas acciones, los asentamientos y barrios populares se ven favorecidos al aumentar el valor del suelo y la introducción de servicios públicos como agua, drenaje, etc. Sin embargo, como mencionan Sabatini et al., (2001):

"Los grupos pobres cercanos se favorecen por la llegada de estos desarrollos, tanto en términos objetivos (trabajo, servicios, equipamiento urbano), como subjetivos (sentimiento de pertenecer a un área que está progresando) (...) No obstante la existencia de las rejas y muros dan paso a la segregación" (p.29).

Bajo este discurso es que muchas veces el Estado se vale para permitir que las desarrolladores inmobiliarios lleven adelante el "progreso" que va en contra de la misma lógica del urbanismo. Por tanto, es necesario que el Estado impulse políticas habitacionales que no solo tengan como objetivo reducir el déficit habitacional, sino también, combatir la división social y espacial del hábitat urbana mediante políticas de integración social que permitan un buen desarrollo urbano. Se debe entonces cambiar la actitud de evitar tener en cuenta el proceso de gentrificación en la antigua área central de la ciudad, producida por las intervenciones urbanas. El realizar un recorrido por la historia de la ciudad, se tiene que las actuaciones no han sido planificadas, ni 
ejecutadas de forma correcta que lleven a crear una ciudad consolidada. La carencia de gestión y planificación responden a intereses monetarios-inmobiliarios, y no en busca del desarrollo urbano de la ciudad.

\section{Conclusión}

Latinoamérica posee casi la mitad de las ciudades dentro del tamaño intermedio (48.1\%), lo que invita a la reflexión de cómo se inserta esta relación rango-ciudad en el medio global imperante, y más aún, considerando los acelerados cambios socio espaciales que han experimentado los centros urbanos de toda escala durante el presente siglo. Si a ello se le suma la permanente actividad de eventos naturales catastróficos, se tiene como resultado, suelos urbanos ampliamente vulnerables, tanto a la reconstrucción física como socio económica que se llevan adelante posterior a desastres como terremotos y maremotos. Por lo tanto, la revisión de casos concretos aparece aún más urgente cuando los cambios físicos y sociales que muestran barrios históricos pueden ser explicados a través de la gentrificación.

El caso de Managua revisado no escapa a esta dinámica; el terremoto de 1972 fue claramente un detonante de la gentrificación, lo cual ha sido revisado en su casco histórico. La importancia otorgada por los especialistas miembros de la escuela marxista a los aspectos económicos del suelo es una constante en las investigaciones urbanas, que se ve fuertemente evidenciada dentro de las últimas cuatro décadas analizadas, debido a la especulación inmobiliaria que ha favorecido a un pequeño sector económico vinculado a grupos específicos como es la familia Somoza. Aún más, en la actualidad, el desarrollo de la ciudad Managua ya tiene expresiones propias, como la gentrificación "periférica" que es evidente en la zona sur de la ciudad con el incremento de nuevas áreas residenciales que van desplazando a los pobladores de la zona, transformando el uso de suelo y tipologías arquitectónicas del sitio.

La presente investigación entrega las primeras pinceladas a algunos de los debates teóricos acerca de la gentrificación contemporánea y sus tipos y procesos, así como evidencia que la conformación socio espacial de la antigua área central de Managua y, de la ciudad en general, ha estado determinada por eventos naturales, aspectos económicos, conflictos sociales y políticos (guerras), decisiones políticas a nivel nacional y local, falta de gestión y planificación urbana integral vinculada a la participación ciudadanía. Lo anterior sugiere que la actual conformación de la ciudad evidencia una correlación de múltiples factores propios de la gentrificación urbana y mayor, aun considerando que el terremoto de 1972 ha detonado procesos de reconstrucción muy especulativa que se alejan de las etapas que deberían seguir a un evento catastrófico, revisado por el Diagrama de Ciclo de Alerta del Terremoto (DCAT).

Es evidente la gentrificación como resultado de la correlación de un conjunto de elementos que se conjugan para dar paso a este proceso urbano, el cual sumado a otros aspectos, han vuelto a Managua una ciudad dispersa, económicamente costosa, tanto para los pobladores como para la municipalidad. Ello además incide en la falta de identidad y de espacios de encuentro y esparcimiento donde, por el contrario, cada vez más aumentan los asentamientos humanos espontáneos, muchas veces debido a la carencia de coordinación a nivel local (ALMA) y estatal (Gobierno Central) a la hora de realizar las intervenciones, lo cual genera contradicciones urbanísticas y sociales. Además de la falta de equipos multidisciplinarios para la realización de políticas, planes, programas y proyectos integrales que lleven a la creación de una ciudad armónica.

La diferenciación de la división social espacial y el proceso de gentrificación resulta de la aplicación "deliberada" de políticas de desarrollo urbano, que en efecto, todo parece indicar que las autoridades han renunciado a administrar la ciudad en beneficio de todos sus pobladores. Ello ha llevado a una ciudad fragmentada, segregada, con exclusión y desintegración socialmente, 
que más atomizada y menos jerarquizada, ha ido sustituyendo a la ciudad orgánica y compacta de antes del terremoto de 1972. Por lo tanto, es necesario examinar el desplazamiento de residentes tradicionales como parte del proceso de gentrificación y, que según algunos teóricos, representa su consecuencia más importante. Los efectos negativos de la gentrificación son asumidos por la población que es expulsada fuera de la localidad por el desarrollo de esta dinámica, causante de la des-configuración y pérdida del dominio del territorio que habitaron por décadas. En este desplazamiento se involucran dos elementos: el tangible, con la salida de la población residente; y el simbólico, relacionado con problemáticas de exclusión social y falta de integración al proceso de renovación urbana.

Con el caso estudiado de la antigua aérea central y el resto de la ciudad de Managua, se quiere poner sobre la mesa la reflexión de cómo un fenómeno natural (terremoto) que afecta a una ciudad, podría ser visto como una oportunidad para restablecer el ciclo natural de políticas e intervenciones urbanas adecuadas para el crecimiento de las ciudades. La adecuada gestión y planificación urbana puede generar ciudades sostenibles y urbanamente coherentes, y así conseguir la reducción de los procesos de gentrificación urbana.

\section{Agradecimientos}

Los autores agradecen al proyecto Urban_Managua y la Universidad de Chile por permitir la visita del profesor Néstor Saúl López Irías en Santiago de Chile en el mes de junio 2014 y, con ello, realizar un análisis de contraste para la investigación sobre Gentrificación en ciudades intermedias de Chile y Nicaragua.

\section{Referencias}

ALEXANDER, D. E. (2012). 'Disasters: lessons learned?' Journal of Geography and Natural Disasters 2(1): $1-2$.

ARIZAGA, D. (2003). 'Recuperación de las Áreas Centrales', Gestión Urbana para el Desarrollo Sostenible en América Latina y el Caribe June V: 203-242. Santiago: Comisión Económica para América Latina y el Caribe CEPAL.

ARRIAGADA C., J. MORENO and E. CARTIER (2007). Evaluación de Impacto del Subsidio de Renovación Urbana en el Área Metropolitana del Gran Santiago 1991-2006, Política Habitacional y Planificación, VII: 327. Santiago, Chile: DITEC, MINVU.

ATKINSON, R. (2000). The hidden costs of gentrification: displacement in central London. Journal (15), 307-326.

BARAHONA, M. (1985) El crecimiento de la ciudad de Managua. Alcaldía de Managua. Managua, Nicaragua.

BECERRIL-SÁNCHEZ, T.; J. MÉNDEZ y C. GARROCHO (2013). Urbanizaciones cerradas y transformaciones socio espaciales en Metepec, Estado de México, Eure 39, 117: 191-213.

BELLET, C. and J.M. LLOP (2002). Intermediate Cities. Profiles and Agenda, Second Phase of the UIACIMES Programe Intermediate cities and world urbanisation. Lleida, Spain: Ajuntament de Lleida.

BID (2014) Plan de acción: Managua sostenible-Iniciativa ciudades emergentes y sostenibles. Managua, Nicaragua.

BOLAY, J-C. and A. RABINOVICH(2004). 'Intermediate cities in Latin America risk and opportunities of coherent urban development.' Cities21(5): 407-421

CALDERA SANCHEZ, A. (2012). 'Building Blocks for a Better Functioning Housing Market in Chile.' OECD Economics Department Working Papers, No. 943, OECD Publishing. Available: http://dx.doi.org/10.1787/5k9fj3hgsnvh-en. 
CALDEIRA, T. (2000). City of Walls: Crime, Segregation, and Citizenship in São Paulo. London; Berkeley: University of California Press.

CAMARENA LUHRS, M. R. (1987). Grandes rutas del espacio social. Elementos para el análisis político de las direcciones recientes de la movilidad en México. Tesis de Doctorado, Facultad de Ciencias Políticas y Sociales (UNAM), México, 322 pp.

CEPAL Comisión Económica para América Latina (2010). Terremoto en Chile Una primera mirada al 10 de marzo de 2010. Santiago, Chile: Naciones Unidas.

CONTRERAS. Y, 2011. 'La recuperación urbana y residencial del centro de Santiago: Nuevos habitantes, cambios socioespaciales significativos' Eure 37(112): 89-113.

DAVIDSON, M. and L. LEES 2010. 'New-Build Gentrification: Its Histories, Trajectories, and Critical Geographies 'Population, Space and Place 16: 395-411.

DE MATTOS C., C. RIFFO, G. YAÑEZ, and X. Salas (2005) 'Reestructuración del Mercado Metropolitano de Trabajo y Cambios Socio Territoriales en el Gran Santiago', Final Report Fondecyt Project 1040838. (Santiago: IEU - PUC, INE).

DESENA, J. (2009). Gentrification and inequality in Brooklyn. New York: Lexington Books.

EDUM Esquema de Desarrollo Urbano de Managua (1982). Alcaldía de Managua.

GASIC, I. (2013) Gentrificación en el peri-centro metropolitano del gran Santiago. Escuela de Geografía, Universidad de Chile.

HAASE, A., S. KABISCH, A. STEINFÜHRER, A. BOUZAROVSKI, R. HALL and P. OGDEN (2010). 'Emergent Spaces of Reurbanisation: Exploring the Demographic Dimension of Inner-city Residential Change in a European Setting' Population, Space Place 16, 335-343.

HARDY, S. (2000). Managua: entre la fragmentación urbana y los riesgos naturales. Revista de historia $N^{\circ}$ 15 - 16. Instituto de Historia de Nicaragua y Centroamérica.

HAYDEN D, (1995).The Power of Place: Urban Landscapes as Public History (Cambridge, MA: MIT Press). HERZER, H. (2008). Con el corazón mirando al sur: Transformaciones en el sur de la ciudad de Buenos Aires. Buenos Aires: Espacio Editorial.

HIDALGO, R. (2004) 'De los Pequeños Condominios a la Ciudad Vallada: Las Urbanizaciones Cerradas y la Nueva Geografía Social en Santiago de Chile (1990-2000)',EURE.30, December 91: 29-52. Printed versionISSN 0250-7161.

INIDE, Instituto Nacional de Información de Desarrollo (2005). Managua, Nicaragua.

INZULZA, J. (2014). Gentrification in the context of post-earthquake reconstruction urban policies: a review of the Chilean experience, Global Urban Research Centre Working Paper 9, The University of Manchester, ISBN: 978-1- 907120-08-4.

INZULZA, J. (2012) Latino gentrificación? Focusing on Physical and Socioeconomic Patterns of Change in Latin American Inner Cities, Urban Studies.

IRP International Recovery Platform (2007). A Strategic Framework. Available: www.recoveryplatform.org/.

JANOSCHKA, Michael; SEQUERA, Jorge and SALINAS, Luis (2014). Gentrification in Spain and Latin America - a Critical Dialogue. International Journal of Urban and Regional Research38 (4), 1234-1265.

JONES, G. and A. VARLEY (1999) 'The Reconquest of the Historic Centre: Urban Conservation and Gentrification in Puebla, Mexico', Environment and Planning31:1547-1566.

JONES, G. and P. WARD (2004) 'The End of Public Space in the Latin American City?', Memoria of the Binational Conference. The Mexican Center of LILAS. University of Texas at Austin, March 4-5. 
KEARNS, A., E. WHITLEY, P. MASON and L. BOND (2012). 'Living the High Life'? Residential, Social and Psychosocial Outcomes for High-Rise Occupants in a Deprived Context' Housing Studies 27 (1): 97-126.

LAWNER, M. (2010). Carta Abierta al Presidente Piñera, Revista de Urbanismo 22. Available: http://www.revistas.uchile.cl/index.php/RU/article/viewArticle/6384/8649.

LEES, L, T. SLATER, and E. WYLY 2008. Gentrification. New York \& London: Routledge.

LÓPEZ, N. \& SUÁREZ, B. (2013). Segregación Urbana en Managua: Estudio de segregación socioresidencial en la ciudad de Managua. (Tesis inédita para optar al grado de Sociólogo). UCA, Nicaragua.

LOZANOVKSA, M. (2012). 'Kenzo Tange's Forgotten Master Plan for the Reconstruction of Skopje, Fabrications' The Journal of the Society of Architectural Historians, Australia and New Zealand 22(2): 140163.

LUKIĆ, I. (2011). 'Relation between creativity and planned regulation in the process of shaping urban skyline' Limes: Borderland Studies 4(2): 131-149.

LYNCH, K. (1960) The Image of the City (Cambridge: M.I.T. Press).

MANSILLA, E. (2011). Riesgo Urbano y Políticas Públicas en América Latina: La Irregularidad y el Acceso al Suelo in GAR, Global Assessment Report on Disaster Risk Reduction.

MAURRASSE, D. (2006). Listening to Harlem: gentrification, community and business. London: Routledge. MITLIN, D. and D. SATTERTHWAITE (2013). Urban Poverty in the Global South. Scale and Nature. London and New York: Routledge.

MOSER, C., G. SOU, and A. STEIN (2011). Climate change and assets, Briefing Paper 2, Global Urban Research Centre, University of Manchester.

OLIVER-SMITH, A. (1994). Reconstrucción después del desastre: una visión general de secuelas y problemas, in Al Norte del Rio Grande, Ciencias sociales, desastres: una perspectiva norteamericana, edited by A. Lavell. Santiago: Red de Estudios Sociales en Prevención de Desastres en América Latina.

OLSHANSKY, R. and S. CHANG (2009). 'Planning for disaster recovery: emerging research needs and challenges' Progress in Planning 72: 195-250.

ONESTINI, M. (2011). 'Water Quality and Health in Poor Urban Areas of Latin America' International Journal of Water Resources Development 27(1): 219-226.

OZERDEM, A. and G. RUFINI (2013). 'L'Aquila's reconstruction challenges: has Italy learned from its previous earthquake disasters?' Disasters37 (1): 119-143.

PLAN REGULADOR DE ÁREA CENTRAL DE LA CIUDAD DE MANAGUA (1982). Alcaldía de Managua. Managua, Nicaragua.

PLANES PARCIALES DE ORDENAMIENTO URBANO DE LA CIUDAD DE MANAGUA (2000-2006). Alcaldía de Managua. Managua, Nicaragua.

PNUD, Programa de las Naciones Unidas para el Desarrollo (2010). Evaluación del Riesgo de Desastres, Buró de Prevención de Crisis y Recuperación. Available:

http://www.undp.org/content/undp/es/home/librarypage/crisis-prevention-and-recovery/disaster_risk_assessment.html.

PNUD Programa de las Naciones Unidas para el Desarrollo (2004). Un informe mundial. La reducción de riesgos de desastres. Un desafío para el Desarrollo, Dirección de Prevención de Crisis y de Recuperación. New York: John S. Swift Co.

POBLETE, J. (2013). Urbanismo para pueblos pequeños.

Availablehttp://www.latercera.com/noticia/opinion/ideas-y-debates/2013/02/895-509539-9-urbanismo-para-pueblospequenos.shtmIRedfern 1997 
RODRÍGUEZ, A. and L. WINCHESTER, (2004). Santiago de Chile: Una Ciudad Fragmentada, in De Mattos C, M E Ducci, A. Rodríguez and G Yañez (eds) Santiago en la Globalización: ¿Una Nueva Ciudad? (Santiago: Ediciones SUR).

ROJAS, E. (2004) Volver al Centro. La Recuperación de las Urbanas Centrales. Rodriguez, E and E. Wegel in Collaboration (Washington, D.C.: Banco Interamericano de Desarrollo).

SABATINI, F.; CÁCERES, G.; y CERDA, J (2001). Segregación residencial en las grandes ciudades chilenas, 1970-1992: Concepción y Valparaíso. Santiago: Mideplan.

SARAVIA, O. 2013. Compendio de fotografías del antiguo centro histórico de la ciudad de Managua. Managua, Nicaragua.

SARGATAL, M. (2000) 'El Estudio de la Gentrificación', Biblio 3W, Revista Bibliográfica de Geografía y Ciencias Sociales, May, 228.

SMITH, N. (1979). Towards a Theory of Gentrification: A Back to the City Movement by Capital, not People. Journal of the American Planning Association 45 (4): 538-548.

SMITH, N. (1996) La nueva frontera urbana Ciudad revanchista y gentrificación. Creative Commons.

SVS Superintendencia Valores y Seguros (2012). Terremoto 2010. Análisis e Impacto del 27-F en el Mercado Asegurador. Santiago, Chile: Andros.

UNITED NATIONS (2002). World Urbanization Prospects. The 2001 Revision. New York: United Nations Population Division.

USGS U.S. Geological Survey (2013). World Earthquake Information by Country/Region, data base. Available in http://earthquake.usgs.gov/earthquakes/world.

WAMSLER, C. (2007).Managing Urban Disaster Risk. Analysis and AdaptationFrameworksforIntegratedSettlementDevelopmentProgrammingfortheUrban Poor, Doctoral thesis $\mathrm{N}^{\circ} 4$ of HDM thesis series. Lund, Sweeden: HDM Lund University.

WEISS FAGEN, P. (2008). Natural disasters in Latin America and the Caribbean: national, regional and international interactions. A regional case study on the role of the affected state in humanitarian action, Humanitarian Policy Group working paper, Georgetown Universityhttp://www.odi.org.uk/hpg/affected_state.html. 